\title{
Control of osmotic pressure to improve cell viability in cell- laden tissue engineering constructs
}

\author{
A. F. Carvalho ${ }^{1,2}$ (I) | L. Gasperini ${ }^{1,2}$ | R. S. Ribeiro ${ }^{1,2}$ | A. P. Marques ${ }^{1,2}$ | R. I. Reis ${ }^{1,2}$
}

${ }^{1} 3$ B's Research Group - Biomaterials, Biodegradable and Biomimetics, Avepark Parque de Ciência e Tecnologia, Zona Industrial da Gandra, Barco - Guimarães, Portugal

${ }^{2}$ ICVS/3B's - PT Government Associate Laboratory, Braga/Guimarães, Portugal

Correspondence

Rui L Reis, Ave Park, Zona Industrial da Gandra, S. Cláudio do Barco, 4805-017 Barco, Guimarães, Portugal.

Email: rgreis@dep.uminho.pt

Funding information

Seventh Framework Programme, Grant/Award Number: FP7-IDEAS-ERC; ERC-2012ADG_20120216-321266 for pr; Fundação para a Ciência e a Tecnologia, Grant/Award Number: SFRH/BPD/109595/2015

\begin{abstract}
Design of tissue engineering strategies deals with the need to balance both biomaterials characteristics and techniques specificities, often resulting in cell-compromising processing conditions. One important factor often disregarded is the osmotic pressure to which cells are exposed. An in-house microfluidic system was used to prove that addition of an osmotic regulator significantly benefits the generation of viable cell-laden hydrogels under harsh processing conditions. Human adipose-derived stem cells were resuspended in 1.5\% alginate and $1 \%$ gellan gum (GG; $w / v)$ solutions containing different concentrations (0.12 M, 0.25 M and $1.5 \mathrm{M})$ of sucrose as osmotic regulator. GG (in water) and alginate (in water or phosphate-buffered saline) solutions were used to vary the conditions under which cells were kept prior processing. Independently of the polymer, addition of sucrose did not affect the processing conditions or the viscosity of the solutions, except at $1.5 \mathrm{M}$. The obtained results clearly demonstrate that inclusion of $0.25 \mathrm{M}$ sucrose during processing of the cell-laden hydrogels allowed to keep cell viability around $80 \%$, in opposition to the $20 \%$ observed in its absence, both for GG and alginate-derived hydrogels prepared in water. Impressively, the level of cell viability observed with the inclusion of $0.25 \mathrm{M}$ sucrose, $76 \%$ for GG and $86 \%$ for alginate, was similar to that obtained with the standard alginate solution prepared in phosphate-buffered saline (82\%). The beneficial effect of sucrose was observed within the first $5 \mathrm{~min}$ of processing and was maintained for prolonged experimental setups with viability values above $50 \%$, even after a 2 -h time-frame and independently of the material.
\end{abstract}

\section{KEYWORDS}

bioprinting, cell viability, cell-laden hydrogels, microfluidics, osmotic regulator, sucrose

\section{1 | INTRODUCTION}

Designing strategies for tissue engineering continuously challenges the application of cell-biomaterial systems in biomedicine. The need for more versatile approaches imposes the identification/optimization of methods that not only address material features and construct design, but also take into account the conditions in which cells are maintained. Attempts to cope with biomaterials characteristics, such as, for example, crosslinking mechanisms (Gasperini, Mano, \& Reis, 2014), and techniques specificities, such as bioprinting (Malda et al., 2013) or microfluidics, often result in cell-compromising processing conditions (Rozman \& Doull, 2000). If cells are maintained for periods that can range from few minutes to even hours in less than ideal conditions, they are subjected to different types of stress stimuli. Mechanical and shear stress, and osmotic pressure, for example, compromise membrane integrity and cell viability, ultimately impacting the performance of the generated construct.

Control of osmotic balance is often achieved by the use of physiological buffers as solvents for the biomaterial. Although these work for several polymers (e.g., alginate) (Wüst, Godla, Müller, \& Hofmann, 2014), the crosslinking mechanism is often a limiting factor. Gellan gum (GG), for example, also crosslinks in the presence of monovalent ions (Oliveira et al., 2010); therefore, water has been the primordial solvent used to work with it. It is expected that cells resuspended in a wateror nonphysiologically buffered-based polymeric solutions (hyposmotic conditions) are subjected to osmotic shock. Therefore, we hypothesized that the use of a chemically inert osmotic regulator, such as sucrose, during cell-compromising processing conditions may contribute to preserve cell membrane integrity and reduce cytotoxicity (Figure 1). To address our premise, cell encapsulation experiments were performed using a 
microfluidics system and two materials with different intrinsic properties, GG and alginate. The effect of the inclusion of sucrose as an osmotic regulator was tested both in mild and extreme conditions, varied in terms of experimental time frame and polymeric solvents.

Alginate (1.5\% w/v; Sigma-Aldrich, Portugal) and GG (1\% w/v; Sigma-Aldrich, Portugal) solutions were prepared respectively in water or phosphate-buffered saline (PBS; Gibco, Thermo Fisher Scientific, Portugal), and water. Sucrose (Sigma-Aldrich, Portugal) was used at hyposmotic $(0.12 \mathrm{M})$, isosmotic $(0.25 \mathrm{M})$ and hyperosmotic $(1.5 \mathrm{M})$ concentrations.

Human adipose-derived stem cells (hASCs) were isolated as previously described (Cerqueira et al., 2013) from lipoaspirate samples from healthy individuals undergoing aesthetic procedures, after informed consent and under a collaboration protocol between Hospital da Prelada (Porto) and the 3B's Research Group, approved by the ethical committees. Briefly, after enzymatic digestion, centrifugation and red blood cell lysis steps, hASCs were obtained by plastic-adherence selection for 48 h. Cells were cultured in a-MEM (Gibco, Thermo Fisher Scientific, Portugal) supplemented with 10\% FBS (Gibco, Thermo Fisher Scientific, Portugal) and $1 \%$ antibiotic/antimycotic (Gibco, Thermo Fisher Scientific, Portugal) and were used between passages 2 and 5 .

An in-house adapted microfluidics system (Gasperini, Marques, \& Reis, 2016) using a Microfluidic chip (Dolomite, UK) coupled to a OB1 pressure controller and a flow sensor (Elvesys, France), was used to obtain cell-laden GG and alginate hydrogel fibres. In detail, $10^{6}$ hASCs were mixed with $1 \mathrm{ml}$ of alginate or GG solution and applied to the microfluidics system. The produced fibres were collected in a $0.1 \mathrm{M} \mathrm{CaCl}_{2}$ (Sigma-Aldrich, Portugal) crosslinking bath and immediately placed in culture medium. Processing timeframe ranged from 5 to $20 \mathrm{~min}$. Cell viability was assessed after $24 \mathrm{~h}$ in culture after calcein-AM (Life Technologies, Thermo Fisher Scientific, Portugal) and propidium iodide (PI; Molecular Probes, Thermo Fisher Scientific, Portugal) labelling. Quantitation was carried out by counting both live and dead cells in 5 independent immunofluorescence images acquired for each condition using Cell Profiler software (Kamentsky et al., 2011). Cells presenting dual staining were considered dead cells.
The effect of the presence of sucrose on the viscosity of alginate and GG solutions was determined by rheological analysis (Rheometer Malvern Kinexus pro+, Malvern, UK). The range of shear rates measured was chosen to cover the shear rates adequate for extrusion processes $(1-100 \mathrm{~Hz})$.

Each experiment was repeated at least 3 times $(n=3)$. Statistical analysis of the different assays was performed using either one-way analysis of variance ANOVA test with Bonferroni's correction method or two-way ANOVA with Tukey's multiple comparison test, with GraphPad Prism®6 software. Percentage of viable cells for each condition is presented with the standard error of the mean (SEM).

Cell viability after 24 hours of culture was significantly compromised in GG hydrogel fibres in the absence of osmotic protector. In comparison, the conditions with $0.12 \mathrm{M}$ and $0.25 \mathrm{M}$ sucrose proved to be advantageous for cell survival. The percentage of viable cells significantly $(p<0.0001)$ increased from $20 \%$ in the absence to $77 \%$ in the presence of $0.12 \mathrm{M}$ and $0.25 \mathrm{M}$ sucrose. Moreover, the hyperosmotic concentration of sucrose $(1.5 \mathrm{M})$ used as control, also positively affected the percentage of viable cells, although not to values above $35 \%$ (Figure 2A). The presence of sucrose in GG solutions did not affect its viscosity, except for the $1.5 \mathrm{M}$ sucrose concentration (Figure 2B).

To understand if the observed effect in osmotic balance, and consequently in cell viability, was sustained for prolonged processing times, a kinetic study was performed. Cells were resuspended in GG water-based solutions in the absence or presence of $0.25 \mathrm{M}$ sucrose (selected form previous experiment) for 5, 10, 20, 60 and 120 min prior processing. The produced cell-laden GG hydrogel fibres were then cultured for $24 \mathrm{~h}$ to assess viability (Figure 2C). Impressively, the presence of the osmotic regulator had an immediate significant $(p<0.0001)$ effect in the improvement of cell viability (from 59 to 83\%), can be seen in the condition corresponding to $5 \mathrm{~min}$ of processing time. It should be noted that cells are in contact with the indicated concentrations of sucrose only prior processing since sucrose is mostly membrane impermeable being then considered as an extracellular osmolyte (Disalvo, 1988; Meryman, 1971). Therefore, diffusion of

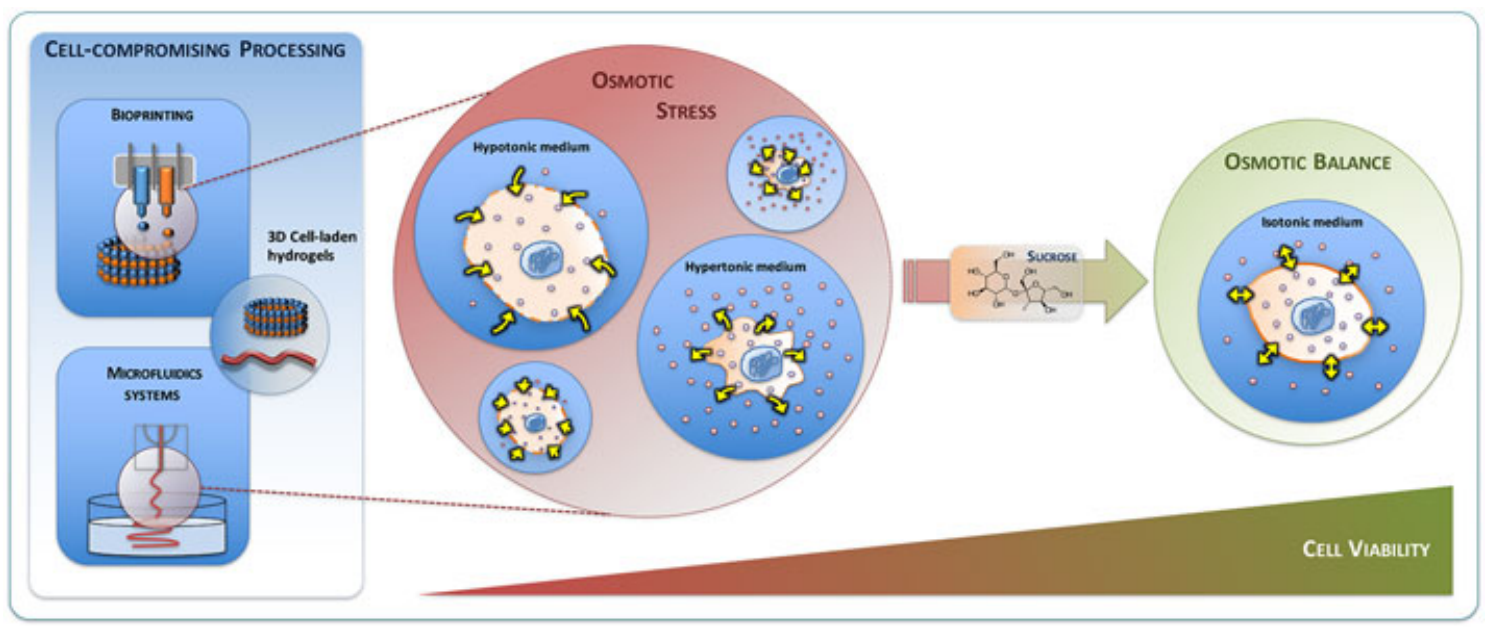

FIGURE 1 Proposed rationale to improve the viability of cell-laden 3D structures for tissue engineering applications processed under cellcompromising conditions such as microfluidics and bioprinting, often subjecting cells to water-based polymeric or nonphysiologically buffered solutions. Osmotic imbalance can lead to cell death either by osmolysis (hypotonic medium) or plasmolysis (hypertonic medium), which can be controlled with the inclusion of inert osmotic regulators such as sucrose during processing [Colour figure can be viewed at wileyonlinelibrary.com] 
(a)
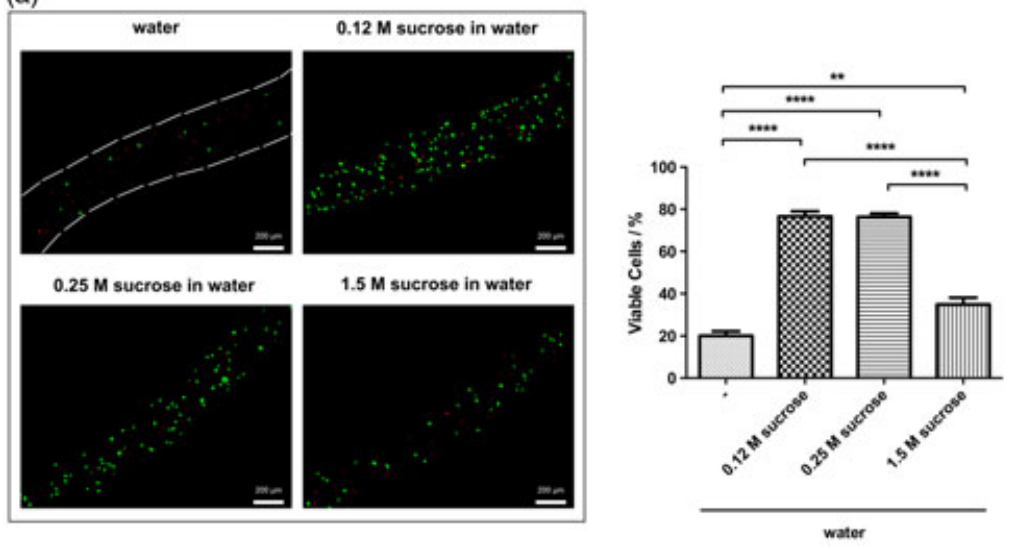

(b)

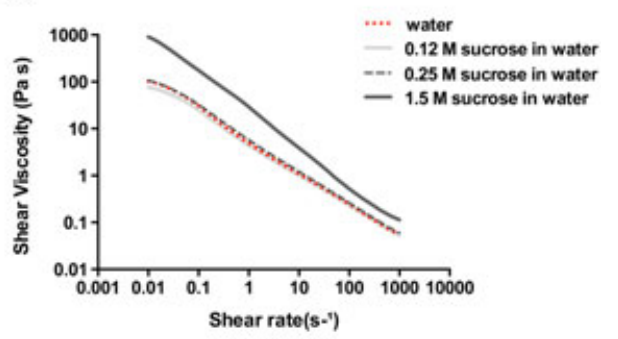

(c)
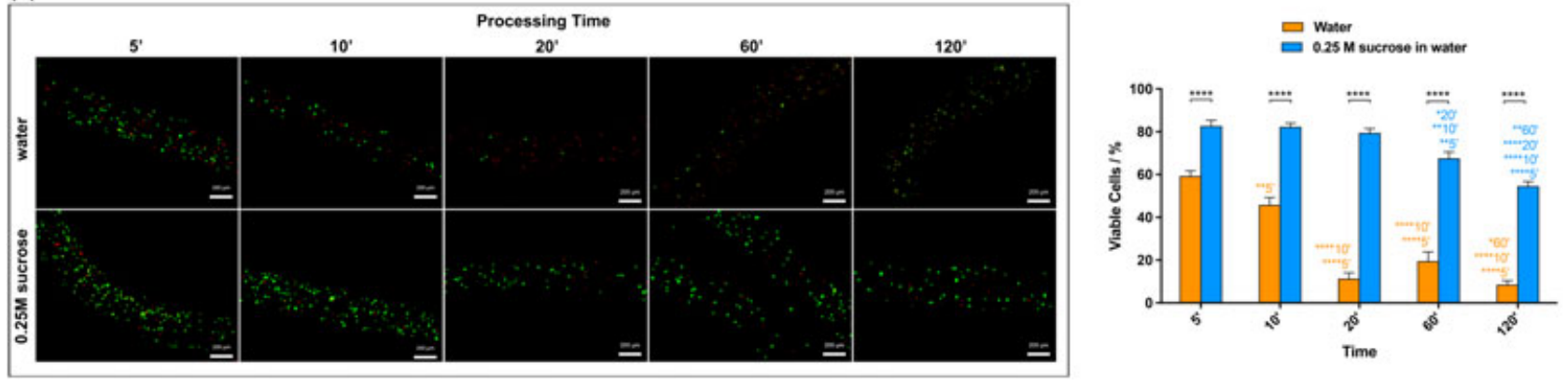

FIGURE 2 Effect of the different concentrations of sucrose ( $0 \mathrm{M}, 0.12 \mathrm{M}, 0.25 \mathrm{M}$ and $1.5 \mathrm{M})$ on the (a) viability of hASCs encapsulated in GG hydrogel fibres and (B) rheological properties of the respective precursor water-based polymeric solutions. (C) kinetics of cell viability of hASCs encapsulated in water-based GG hydrogel fibres in the absence and presence of $0.25 \mathrm{M}$ sucrose, along the processing of the fibres (from 5 min up to $2 \mathrm{~h}$ ). The percentage of viable cells was quantified using cell Profiler software in the fluorescence images of calcein-AM and PI staining the viable (green) and the dead (red) cells. In (a), white dashed lines outline the hydrogels fibres. Scale bars correspond to $200 \mu \mathrm{m}$. Graphs show the mean percentage of viable cells for each condition with SEM, $n=3$. Data were analysed by one-way ANOVA with Bonferroni's multiple comparison test (a) or by two-way ANOVA with Tukey's multiple comparison test (C). ${ }^{*} p<0.05,{ }^{* *} p \leq 0.01,{ }^{* * *} p \leq 0.0001$ [Colour figure can be viewed at wileyonlinelibrary.com]

sucrose from the fibres is expected to occur immediately after immersion in the cross-linking solution and/or in the culture medium (Bayarri, Rivas, Costell, \& Durán, 2001). While in the absence of sucrose cell viability was significantly and successively decreased with the processing time (from 59 to $46 \%$ and to $9 \%$ at 5, 10 and 20 min, respectively), in its presence the positive effect was kept along the processing time and for up to $2 \mathrm{~h}$. Nonetheless, even in the presence of sucrose, after $20 \mathrm{~min}$ of processing the percentage of viable cells diminished from $79.5 \%$ to $68 \%$ and then to $55 \%$, respectively for $1 \mathrm{~h}$ and $2 \mathrm{~h}$.

To be able to compare the effect of sucrose in the improvement of cell viability under physiological conditions, the same set of experiments was conducted with alginate. Although alginate is commonly prepared in PBS, water-based alginate solutions, in the absence or presence of $0.25 \mathrm{M}$ (isosmotic) and $1.5 \mathrm{M}$ (hyperosmotic) sucrose were also considered (Figure 3). In the alginate hydrogels prepared in water in the absence of sucrose cell viability results (17\%) were comparable to those obtained with GG, confirming the harsh effect of hypotonic water-based polymeric solutions. In the presence of $0.25 \mathrm{M}$ sucrose, this value increased to about $86 \%$, similarly to the $77 \%$ obtained with GG in the same isosmotic conditions. Strikingly, the percentage of cell viability in the water-based alginate hydrogels in the presence of $0.25 \mathrm{M}$ sucrose was also comparable to that obtained with PBS, both in the absence and in the presence of $0.25 \mathrm{M}$ sucrose, confirming the osmotic protector role of sucrose in the water-based hydrogels. In the presence of $1.5 \mathrm{M}$ sucrose, the percentage of viable cells was significantly lower ( $p<0.0001 ; 64 \%$ ) than in the presence of $0.25 \mathrm{M}$ sucrose, independently of the solvent, and in the standard PBS condition (Figure 3A).

Alginate and GG have different resistance to flow but both show the typical shear thinning behaviour (decrease of viscosity with higher shear rates; Figure 2B and 3B) of many polymeric solutions (Morris, 1990). At rest, alginate is liquid-like showing a Newtonian plateau while GG is solid-like showing an increasing viscosity, at low shear rates. The flow behaviour of the water- and PBS-based polymeric solutions did not change in the presence of sucrose except for the $1.5 \mathrm{M}$ of sucrose, in agreement with what was previously reported for alginate (Pongsawatmanit, Ikeda, \& Miyawaki, 1999). Nonetheless, the different properties of GG and alginate, in particular the shear viscosity, also impacted the percentage of viable cells observed in the presence of $1.5 \mathrm{M}$ of sucrose. Higher shear viscosity has been correlated with higher osmotic pressure, thus compromising cell viability (Donnan \& Rose, 1950; Loeb, 1921), which justifies the lower percentage of cell viability observed in the water-based GG in comparison to the alginate hydrogels in the hyperosmotic conditions.

To assess if the immediate effect of sucrose changed with the material, the kinetic experiment was performed for the water-based alginate along the same processing time as for the GG. Similarly, cells were protected when re-suspended in a water-based alginate solution 
(a)
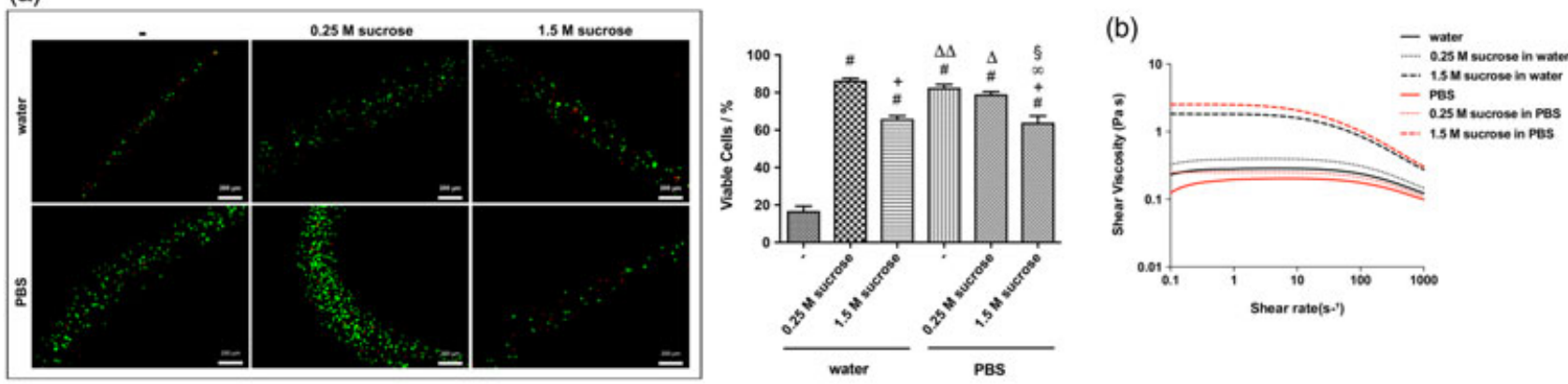

(c)
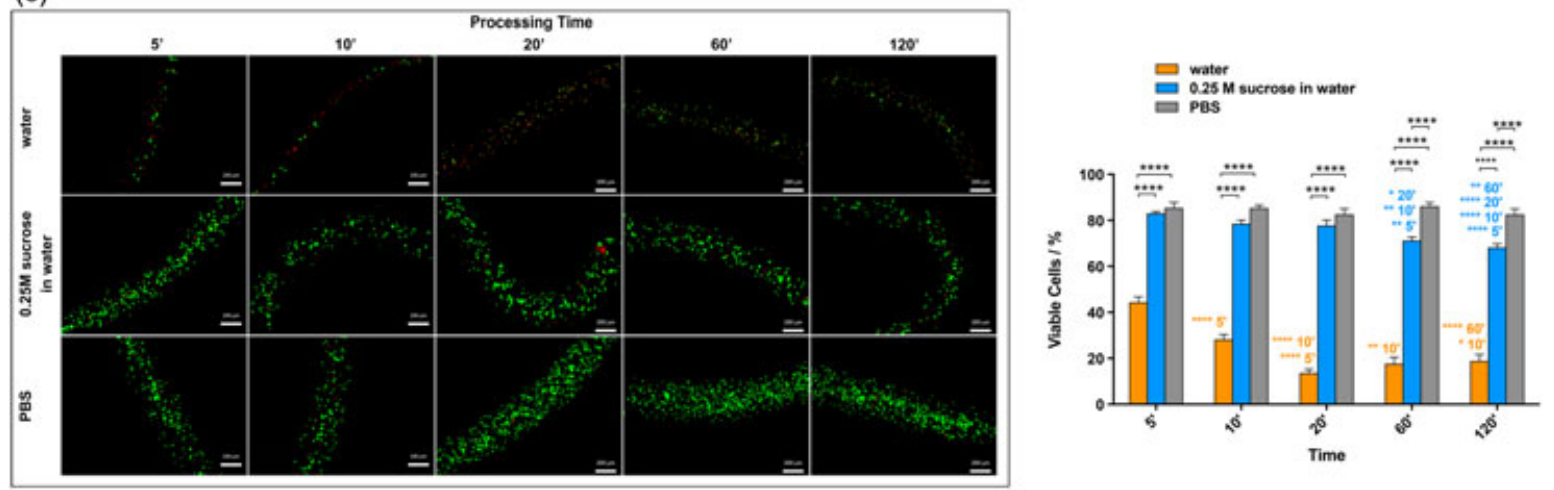

FIGURE 3 Effect of the different concentrations of sucrose $(0 \mathrm{M}, 0.12 \mathrm{M}, 0.25 \mathrm{M}$ and $1.5 \mathrm{M})$ on the (a) viability of hASCs encapsulated in alginate hydrogel fibres and (B) rheological properties of the respective precursor water- and PBS-based polymeric solutions. (C) kinetics of cell viability of hASCs encapsulated in water-based alginate hydrogel fibres in the absence and presence of $0.25 \mathrm{M}$ sucrose, along the processing of the fibres (from 5 min up to $2 \mathrm{~h}$ ). Standard PBS condition was also used as control under the same kinetic conditions. The percentage of viable cells was quantified using cell Profiler software in the fluorescence images of calcein-AM and PI staining the viable (green) and the dead (red) cells. Scale bars correspond to $200 \mu \mathrm{m}$. Graphs depict the mean percentage of viable cells for each condition with SEM, $n=3$. Data were analysed by one-way ANOVA with Bonferroni's multiple comparison test (a) or by two-way ANOVA with Tukey's multiple comparison test (C). \#p < 0.0001 relatively to water only; ${ }^{+} p<0.0001$ relatively to $0.25 \mathrm{M}$ sucrose in water; $\Delta p<0.001, \Delta \Delta p<0.0001$ relatively to $1.5 \mathrm{M}$ sucrose in water; $\infty p<0.0001$ relatively to PBS only; $\S p<0.0001$ relatively to $0.25 \mathrm{M}$ sucrose in PBS. ${ }^{*} p<0.05,{ }^{* *} p<0.01,{ }^{* * * *} p \leq 0.0001$ in between the solvent conditions (water, $0.25 \mathrm{M}$ sucrose in water, PBS) for each time point and in between time points [Colour figure can be viewed at wileyonlinelibrary.com]

containing $0.25 \mathrm{M}$ sucrose. In the shorter processing time, a recovery from $44 \%$ (absence of sucrose) to $83 \%$ was observed. This effect was even more notorious for longer times and up to $20 \mathrm{~min}$, since in the absence of sucrose a successive reduction in cell viability was detected (from 44 to 14\%). Moreover, the recovered cell viability in the presence of sucrose was maintained to levels of $\sim 80 \%$, similar to those observed when alginate was dissolved in the physiological buffer PBS, although only up to 20 min as also shown for GG. Dissolution of both alginate and GG in $0.25 \mathrm{M}$ sucrose clearly benefits cell-encapsulation conditions. However, with prolonged experimental setup, osmotic pressure is not the only cell-compromising stress at play, as it is demonstrated after 20 min of processing time in the water-based GG and alginate solutions with the decrease in cell viability. After this time point, factors such as temperature, $\mathrm{pH}$ and oxidative status also contribute to increase cell stress load and thus impair cell survival (de Nadal, Ammerer, \& Posas, 2011).

Herein, it is demonstrated that sucrose can be used as an osmotic protector in cell-compromising processing conditions. The use of two materials, that have been applied in the development of a wide range of cell-laden hydrogels for tissue engineering and have distinct intrinsic properties, including cross-linking mechanisms, allowed showing the versatility of the approach. Additionally, except for the $1.5 \mathrm{M}$ concentration (hyperosmotic), sucrose did not affect the flow behaviour of the materials, thus confirming its inertness over the processing method. However, processing times longer than 20 min can be significantly detrimental.

The use of other natural or synthetic molecules as osmotic protectors (McGann, 1978) cannot be excluded, as was previously shown for mannose in the development of a new bioink for additive tissue manufacturing (Melchels, Dhert, Hutmacher, \& Malda, 2014). However, the choice of which compound to use must meet the biomaterial- and cell-associated criteria, such as the polymers crosslinking mechanisms and cell viability that were herein considered. This work represents the first approach to further tackle the effect of cellcompromising processing conditions over other cell metabolic functions (Brühlmann et al., 2015; Eagle, Barban, Levy, \& Schulze, 1958), which define the long-term performance of the generated constructs.

\section{ACKNOWLEDGEMENTS}

The authors would like to acknowledge the Portuguese Foundation for Science and Technology (FCT) for personal grant SFRH/BPD/109595/ 2015 under the scope of $\mathrm{POCH}$, co-funded by the European Social Fund and national funds by MCTES. The work developed was supported by the European Research Council (Advanced Grant No. ERC-2012-AdG_20120216-321266 for the project ComplexiTE). 


\section{REFERENCES}

Bayarri, S., Rivas, I., Costell, E., \& Durán, L. (2001). Diffusion of sucrose and aspartame in kappa-carrageenan and gellan gum gels. Food Hydrocolloids, 15(1), 67-73.

Brühlmann, D., Muhr, A., Hemberger, J., Sauer, M., Kornmann, H., Jordan, M., \& Broly, H. (2015). The potential of small molecules to modulate glycosylation by media design. BMC Proceedings, 9(Suppl 9), P38.

Cerqueira, M. T., Pirraco, R. P., Santos, T. C., Rodrigues, D. B., Frias, A. M., Martins, A. R., ... Marques, A. P. (2013). Human adipose stem cells cell sheet constructs impact epidermal morphogenesis in full-thickness excisional wounds. Biomacromolecules, 14(11), 3997-4008.

De Nadal, E., Ammerer, G., \& Posas, F. (2011). Controlling gene expression in response to stress. Nature Reviews Genetics, 12(12), 833-845.

Disalvo, E. A. (1988). Permeability of water and polar solutes in lipid bilayers. Advances in Colloid and Interface Science, 29(1-2), 141-170.

Donnan, F. G., \& Rose, R. C. (1950). Osmotic pressure, molecular weight, and viscosity of sodium alginate. Canadian Journal of Research, 28b(3), 105-113.

Eagle, H., Barban, S., Levy, M., \& Schulze, H. O. (1958). The utilization of carbohydrates by human cell cultures. The Journal of Biological Chemistry, 233(3), 551-558.

Gasperini, L., Mano, J. F., \& Reis, R. L. (2014). Natural polymers for the microencapsulation of cells. Journal of the Royal Society, Interface, 11(100), 20140817.

Gasperini, L., Marques, A. P., \& Reis, R. L. (2016) Microfibers with compartments, their preparation and applications thereof, DP/01/2016. Portuguese national patent 109697.

Kamentsky, L., Jones, T. R., Fraser, A., Bray, M. A., Logan, D. J., Madden, K. L., ... Carpenter, A. E. (2011). Improved structure, function and compatibility for cellprofiler: Modular high-throughput image analysis software. Bioinformatics, 27(8), 1179-1180.

Loeb, J. (1921). The reciprocal relation between the osmotic pressure and the viscosity of gelatin solutions. The Journal of General Physiology, 4(1), 97-112.
Malda, J., Visser, J., Melchels, F. P., Jüngst, T., Hennink, W. E., Dhert, W. J., ... Hutmacher, D. W. (2013). 25th anniversary article: Engineering hydrogels for biofabrication. Adv Mat, 25(36), 5011-5028.

McGann, L. E. (1978). Differing actions of penetrating and nonpenetrating cryoprotective agents. Cryobiology, 15(4), 382-390.

Melchels, F. P. W., Dhert, W. J. A., Hutmacher, D. W., \& Malda, J. (2014). Development and characterisation of a new bioink for additive tissue manufacturing. Journal of Materials Chemistry B Materials for Biology and Medicine, 2(16), 2282-2289.

Meryman, H. T. (1971). Cryoprotective agents. Cryobiology, 8(2), 173-183.

Morris, E. R. (1990). Shear-thinning of "random coil" polysaccharides: Characterisation by two parameters from a simple linear plot. Carbohydrate Polymers, 13(1), 85-96.

Oliveira, J. T., Martins, L., Picciochi, R., Malafaya, P. B., Sousa, R. A., Neves, N. M., ... Reis, R. L. (2010). Gellan gum: A new biomaterial for cartilage tissue engineering applications. Journal of Biomedical Materials Research. Part A, 93(3), 852-863.

Pongsawatmanit, R., Ikeda, S., \& Miyawaki, O. (1999). Effect of sucrose on physical properties of alginate dispersed aqueous systems. Food Science and Technology Research, 5(2), 183-187.

Rozman, K. K., \& Doull, J. (2000). Dose and time as variables of toxicity. Toxicology, 144(1-3), 169-178.

Wüst, S., Godla, M. E., Müller, R., \& Hofmann, S. (2014). Tunable hydrogel composite with two-step processing in combination with innovative hardware upgrade for cell-based three-dimensional bioprinting. Acta Biomateriala, 10(2), 630-640.

How to cite this article: Carvalho A, Gasperini L, Ribeiro R, Marques A, and Reis R. Control of osmotic pressure to improve cell viability in cell-laden tissue engineering constructs. J Tissue Eng Regen Med. 2017;0:1-5. https://doi.org/10.1002/ term.2432 